\title{
Establishment and evaluation of three necrotizing enterocolitis models in premature rats
}

\author{
WEI ZHOU, XIAO-HUI ZHENG, XIAO RONG and LONG-GUANG HUANG \\ Department of Neonatology, Guangzhou Children's Hospital, Guangzhou Women and Children's \\ Medical Center, Guangzhou Medical College, Guangzhou, Guangdong 510623, P.R. China
}

Received May 5, 2011; Accepted August 24, 2011

DOI: $10.3892 / \mathrm{mmr} .2011 .581$

\begin{abstract}
Necrotizing enterocolitis (NEC) is a gastrointestinal disease that usually affects premature infants and has high morbidity and mortality rates. Reliable animal models aid further study of the etiological factors, pathogenesis, prevention and treatment of NEC. The present study aimed to establish NEC models in premature rats using three common methods, and to determine the optimal model establishment method. The study consisted of six groups; in group A, rats were raised with simulated milk and subjected to inhalation of $100 \%$ nitrogen gas (hypoxia) for $90 \mathrm{sec}$, followed by exposure to cold ambient conditions $\left(4^{\circ} \mathrm{C}\right)$ for $10 \mathrm{~min}$ twice daily for 3 days. In group B, rats were exposed to $100 \%$ nitrogen gas for $5 \mathrm{~min}$ and $100 \%$ oxygen for 5 min twice daily for 3 days. Group C rats were intraperitoneally administered $5 \mathrm{mg} / \mathrm{kg}$ lipopolysaccharide. Group D and E rats did not receive any intervention. Group F rats were intraperitoneally administered $1 \mathrm{ml} / \mathrm{kg}$ physiological saline. Groups D-F served as the control groups corresponding to groups A-C, respectively. Following hematoxylin and eosin staining, intestinal tract, liver, lung and kidney tissues were observed under optical microscopy and were scored. Successful NEC induction was measured by a score of $\geq 2$. Rats from groups A-C exhibited reduced movement, abdominal distention, diarrhea, intestinal tractexpansion, and congestion to varying degrees. The pathological scores of intestinal injury in groups A-F were $3.13 \pm 0.64,1.40 \pm 0.52$, $2.00 \pm 0.42,0.30 \pm 0.48,0.30 \pm 0.48$, and $0.40 \pm 0.52$ points, respectively. Significant differences were found between the model groups and their corresponding control groups $(\mathrm{p}<0.01)$. Among the model groups, the histological score of group A was higher than that of groups $B(p<0.01)$ and $C(p<0.05)$. The morbidity rate of NEC in groups A-C was 75, 20 and $50 \%$, respectively. There was no morbidity in groups D-F.
\end{abstract}

Correspondence to: Professor Wei Zhou, Department of Neonatology, Guangzhou Children's Hospital, Guangzhou Women and Children's Medical Center, Guangzhou Medical College, Guangzhou, Guangdong 510623, P.R. China

E-mail: zhouwei_pu002@yeah.net

Key words: premature rat, necrotizing enterocolitis, animal model, evaluation
Compared with groups A and B, injury to the liver, kidney and lung was more severe in group C. Similar symptoms were not observed in groups D-F. Compared with methods of simple hypoxia-reoxygenation or intraperitoneal administration of lipopolysaccharide, the combination of artificial feeding and hypoxia plus cold stimulation most resembles the pathological causes of neonatal NEC. This method resulted in high morbidity, reproducibility and specificity, and was therefore considered an ideal model for establishing NEC.

\section{Introduction}

Necrotizing enterocolitis (NEC) is a gastrointestinal disease that results in high morbidity and mortality during the neonatal period. It occurs at a frequency of 0.3-2.4 per 1000 newborns, and $70 \%$ of cases occur in infants born at a gestational age of $<36$ weeks (1). More specifically, premature infants with birth weights $<1500 \mathrm{~g}$ (very low birth weight, VLBW) are at the greatest risk and account for 70-90\% of all cases of NEC $(1,2)$. With improved perinatal care and application of pulmonary surfactants, many premature infants now survive, although the morbidity rate of NEC remains high $(3,4)$. Although NEC has been greatly studied, and pertinent etiological factors including premature delivery, artificial feeding, hypoxia and bacterial colonization have been identified, the precise pathological causes and pathogenesis remain poorly understood. Reliable animal models may provide a greater understanding of the pathological causes, pathogenesis, prevention and treatment of NEC.

No experimental models of spontaneous NEC are currently available. Therefore, numerous methods and a variety of species have been employed to build an experimental NEC model over the past decades. Rats, mice and pigs have all been used to study NEC; however, rats were most frequently used to build the NEC model. Rats have a number of special advantages; for example, rats are more cost-effective than pigs and non-human primates, and the gestation period of rats is also shorter. Second, although rats differ from humans in a variety of ways, including their developmental, anatomical and physiological characteristics, they are highly related in terms of the similarity of genes and biochemical pathways $(5,6)$. A further and extremely significant point is that rats are born relatively immature with respect to the stage of gut development; the immature rat gut bears a closer resemblance to that of a human infant born prematurely (6). 
Hypoxia-reoxygenation was frequently used to establish the model of intestinal injury $(7,8)$. The injection of certain inflammatory mediators such as lipopolysaccharide (LPS), platelet-activating factor (PAF) and tumor necrosis factor- $\alpha$ (TNF- $\alpha$ ), with or without superior mesenteric artery clamping, also replicates the intestinal injury to resemble that of NEC (9-12). However, since Barlow et al (13) developed the earliest NEC model, in which the disease was induced through the combined treatment of formula gavages with intermittent episodes of hypoxia and cold stress, an increasing number of similar methods appeared in press (14-16). To compare the various strengths and weaknesses of experimental approaches that have been used, we established NEC rat models using the most commonly used methods, and determined the most optimal model.

\section{Materials and methods}

Experimental grouping. The study was approved by the Ethics Committee of Guangzhou Children's Hospital, China. Twelve specific pathogen-free, pregnant rats were purchased from the Laboratory Animal Center of Guangzhou University of Traditional Chinese Medicine. At 21 days into gestation, the premature rats, weighing 4-7 $\mathrm{g}$, were removed by laparotomy and were assigned to one of 6 groups ( $\mathrm{n}=10$, respectively), which received treatments as follows. Group A rats were raised with simulated milk and periodically subjected to hypoxia and cold stimulation. Group B rats were exposed to $100 \%$ nitrogen gas for $5 \mathrm{~min}$ and $100 \%$ oxygen for $5 \mathrm{~min}$. Group C rats were intraperitoneally administered physiological saline-diluted $5 \mathrm{mg} /$ $\mathrm{ml}$ LPS ( $5 \mathrm{mg} / \mathrm{mg}$ ) (Sigma, St. Louis, MO, USA). Group B and $\mathrm{C}$ rats were returned to their mothers following surgery. Rats in groups D and $\mathrm{E}$ did not receive any intervention. Group $\mathrm{F}$ rats were intraperitoneally administered $1 \mathrm{ml} / \mathrm{kg}$ physiological saline. Groups D-F served as the control groups corresponding to groups A-C, respectively.

Mating and abdominal delivery. A considerable number of adult male rats (weight 390-420 g) and female rats (weight 225-260 g) were selected and mated at 18:30-19:30 every day at a ratio of 1:2. The vaginal smears were observed at 8:30-9:30 of the second day. Rats were identified as having mated if sperm was found in the smears, and this day was considered day zero when calculating the female's gestation period (completed by the Laboratory Animal Center, Guangzhou University of Traditional Chinese Medicine). Following ether anesthesia, rats at 21 days of gestation were fixed to the surgery table to remove premature rats under sterile conditions.

Milk substitutes and artificial feeding. Following the approach reported by Auestad et al (17), every $100 \mathrm{ml}$ milk substitute included $3.2 \mathrm{~g}$ low-birth-weight infant formula (Nestel, Beijing, China), 9.2 g protein powder (Abbott, Shanghai, China) and $44.5 \mathrm{ml}$ intralipid injection (Sino-Swed Pharmaceutical, Beijing, China), which had a total heat of approximately $581 \mathrm{~kJ}$. The final composition and heat is shown in Table I. Following the instructions of Le Mandat Schultz et al (16), the rats were fed $0.15 \mathrm{ml}$ simulated milk by oral intubation using a hand-made gavage tube (intubation depth 2.5-3.0 cm) starting at $2 \mathrm{~h}$ after birth with intubation every $4 \mathrm{~h}$ after that, and then
Table I. Rat milk and simulated milk ingredients.

\begin{tabular}{lcc}
\hline Ingredient & Rat milk & Simulated milk \\
\hline Fat $(\mathrm{g} / \mathrm{l})$ & $108-112$ & 110.0 \\
Protein $(\mathrm{g} / \mathrm{l})$ & $73-77$ & 74.2 \\
Carbohydrate $(\mathrm{g} / \mathrm{l})$ & $23-27$ & 26.5 \\
Calories $(\mathrm{MJ} / \mathrm{l})$ & $5.78-5.92$ & 5.81 \\
\hline
\end{tabular}

adding $0.2 \mathrm{ml}$ each time in the second day, with continuous feeding for 3 days.

Hypoxia and cold stimulation. Premature rats were placed in a hypoxia box with nitrogen gas controlled to $10 \mathrm{l} / \mathrm{min}$. Following a $90-\mathrm{sec}$ absence of oxygen, as detected by a TED-60T oxygen analyzer (Teledyne Technologies, CA, USA), the rats were removed and immediately exposed to cold ambient conditions $\left(4^{\circ} \mathrm{C}\right)$, and subsequently returned to the incubator. This procedure was performed twice daily (at 9:00 and 21:00) for 3 consecutive days, after which the premature rats were placed into the incubators (Yiheng Instruments, Shanghai, China).

Hypoxia-reoxygenation. Premature rats were exposed to 10 1/min nitrogen gas. Following a 5-min complete absence of oxygen, as detected by the TED-60T oxygen analyzer, the rats were exposed to $5 \mathrm{~min}$ of $100 \%$ oxygen and subsequently returned to their mothers. This procedure was performed twice daily for 3 consecutive days; the premature rats were subsequently returned to their mothers each time.

Sample collection. Rats from groups A-C were sacrificed $12 \mathrm{~h}$ after the last operation. Terminal ileal segments, liver, kidney and lung tissues were harvested and fixed in $10 \%$ formaldehyde solution. Specimens from groups D-F were harvested at the corresponding time points.

Tissue pathological examination. Following $10 \%$ formaldehyde fixation, paraffin embedding, coronal sectioning, and hematoxylin and eosin staining, morphological changes were observed under light microscopy. In accordance with previously described pathological scoring criteria (16), intestinal tissue injury was graded. Briefly, intact villi received a score of 0 , sloughing of epithelial cells on villous tips was assigned a score of 1 , and mid-villous damage was scored as 2 . A NEC score of 3 was assigned to sections with complete villous necrosis, and 4 indicated transmural necrosis. The degree of intestinal injury was determined according to the highest points obtained, i.e., $\geq 2$ points indicated successful NEC induction.

Statistical analysis. ata were statistically analyzed using SPSS 13.0 software (SPSS, IL, Chicago, USA) and were expressed as the mean $\pm \mathrm{SD}$. The analysis of variance was employed to compare the groups. A homogeneity test for variance was initially performed. If the variances were normally distributed, the q-test was used to compare the groups. $\mathrm{P}<0.05$ was considered to be statistically significant. 
Table II. Comparison of premature rat body weight and pathological scoring before and after experimentation $(\bar{x} \pm s)$

\begin{tabular}{lcccc}
\hline Group & $\mathrm{n}$ & Before experimentation $(\mathrm{g})$ & After experimentation $(\mathrm{g})$ & Pathological score \\
\hline A & 8 & $5.87 \pm 0.21$ & $-0.32 \pm 0.56^{\mathrm{a}}$ & $3.13 \pm 0.64^{\mathrm{a}}$ \\
B & 10 & $5.58 \pm 0.32$ & $1.86 \pm 0.14^{\mathrm{a}}$ & $1.40 \pm 0.52^{\mathrm{ab}}$ \\
C & 8 & $5.74 \pm 0.99$ & $-0.11 \pm 0.03^{\mathrm{a}}$ & $2.00 \pm 0.42^{\mathrm{ac}}$ \\
D & 10 & $5.81 \pm 0.42$ & $2.52 \pm 0.11$ & $0.30 \pm 0.48$ \\
E & 10 & $5.59 \pm 0.47$ & $3.54 \pm 0.30$ & $0.30 \pm 0.48$ \\
F & 10 & $5.39 \pm 0.34$ & $1.07 \pm 0.15$ & $0.40 \pm 0.52$ \\
\hline
\end{tabular}

${ }^{a} \mathrm{P}<0.01$ vs. corresponding control group (group D corresponded to group $\mathrm{A}, \mathrm{E}$ to $\mathrm{B}$, and group $\mathrm{F}$ to $\mathrm{C}$ ). ${ }^{\mathrm{b}} \mathrm{P}<0.01$ vs. group $\mathrm{A}$. ${ }^{\mathrm{c}} \mathrm{P}<0.05$ vs. group $\mathrm{A}$.

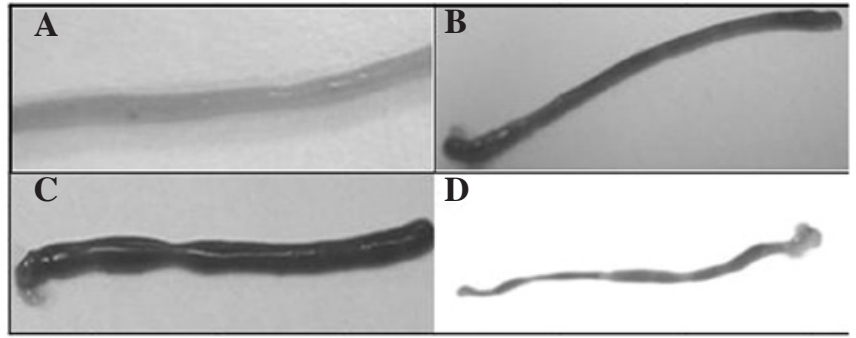

Figure 1. (A) Macroscopic appearance of the gut. Normal intestine (no colour change, no dilatation). (B) Slight dilatation, slightly congested; (C) Extensive dilatation, appeared black. (D) Severe necrosis, extensive discoloration.

\section{Results}

General data and growth status. Following NEC induction, group A rats exhibited reduced movement, slow response times and loss of weight accompanied by abdominal distention, diarrhea, green stool with mucous, and bloody stool. During NEC induction, group B rats presented with dyspnea and convulsion, as well as urinary and fecal incontinence. Immediately following LPS administration, group C rats exhibited trembling, followed by lowered consciousness and breathlessness; 2-3 h later, abdominal distention, diarrhea, and loose green stool were observed. In addition, food intake and movement were reduced, as well as body weight. Groups D-F rats exhibited good movement, normal food intake and defecation, no abdominal distention or diarrhea, and body weight was increased. Body weight changes in premature rats from each group are shown in Table II. Significant differences were observed in weight change between groups A-C and the corresponding groups $\mathrm{D}-\mathrm{F}(\mathrm{p}<0.01)$.

Intestinal tract and pathological changes in the ileum. In group A, the intestinal tract exhibited varying degrees of expansion, congestion and pneumatosis intestinalis. The intestinal tract appeared purple or black. In group B, the intestinal tract was slightly expanded, slightly congested and elastic. In group C, the majority of the intestinal tract exhibited peritoneal dropsy and poor elasticity. The tract was expanded and contained congested intestinal canals. In groups D-F, the intestinal tract was elastic; the jejunum was ivory white and the ileocolon was light yellow (Fig. 1).
Following hematoxylin and eosin staining, ileocecal sections from groups D-F revealed complete ileal structure, intact villi or slightly injured top regions, regularly arranged intestinal glands, no expansion or inflammatory infiltration of vessels in the lamina propria and submucous layer, and a complete muscular layer. The ileocecal sections from groups A-C exhibited varying degrees of pathological changes, including ablated, necrotic villi, loss of intestinal glands and intestinal perforation (Fig. 2).

Pathological grading among the groups. Significant differences were found in intestinal tract injuries among groups A-C and the corresponding groups $\mathrm{D}-\mathrm{F}(\mathrm{P}<0.01)$. Intestinal tract injury was more severe in group $\mathrm{A}$ than groups $\mathrm{B}(\mathrm{P}<0.01)$ and $\mathrm{C}(\mathrm{P}<0.05)$. Pathological scoring of intestinal tract injury and comparison among groups are shown in Table II.

Pathological changes in significant organs. In group C, lung tissue was expanded with congested blood capillaries and severe neutrophil infiltration accompanied by hemorrhage. The liver tissue exhibited inflammatory cell infiltration and hepatocyte hemorrhage, and some hepatocytes presented with dot-shaped necrosis. The kidney tissue was degenerated, with hydropic renal tubular epithelial cells and hemorrhagic interstitium (Fig. 3). Pathological injury included hemorrhage and liver necrosis, and injury to kidney and lung tissues was more severe in groups A and B than group C. No obvious pathological changes were observed in groups $\mathrm{D}, \mathrm{E}$ or $\mathrm{F}$.

NEC morbidity and mortality rates. NEC morbidity rates in groups A, B and C were 75.0\% (6/8), 20.0\% (2/10) and 50.0\% $(4 / 8)$, respectively, whereas no morbidity occured in groups D-F (0/10). Two rats died in groups A and C, respectively, resulting in a mortality rate of $20.0 \%(2 / 10)$. All of the rats survived in the remaining groups.

Analysis of deceased rats. Pathologically, the deceased group A rats exhibited expanded intestinal tracts and thinned purple and black intestinal walls with pneumatosis and lack of elasticity, which corresponded to a pathological score of 4 points. The deceased group $\mathrm{C}$ rats exhibited mild intestinal tract lesions, with a pathological score of 2 points. However, hemorrhagic necrosis of the liver and lungs was extremely severe. 


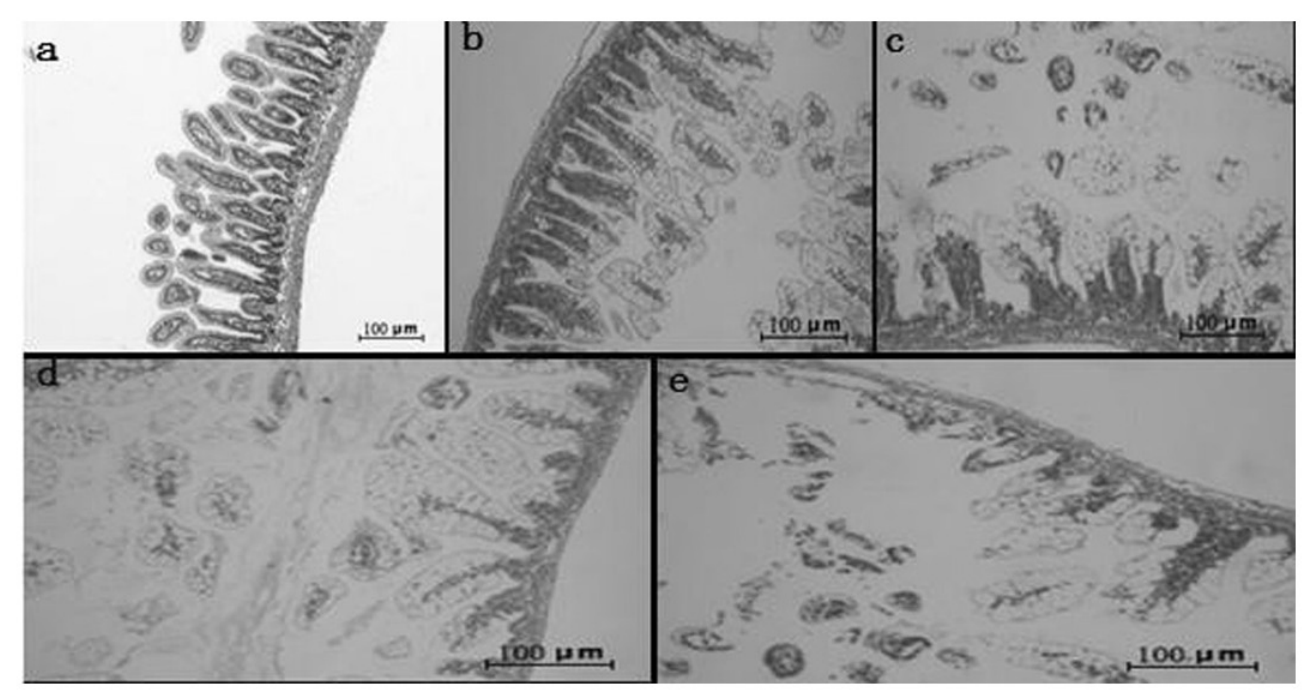

Figure 2. Pathological changes in ileocecal sections (hematoxylin and eosin; magnification, x200). (A) 0 points, complete intestinal mucosal villi with normal structure. (B) 1 point, slightly hydropic villi and epithelial shedding at the top of the villi. (C) 2 points, injury and necrosis in the middle section of the villi. (D) 3 points, loss of villi and identifiable recessus; (E): 4 points, complete loss of mucosal epithelial structures or necrotic mucosal walls.

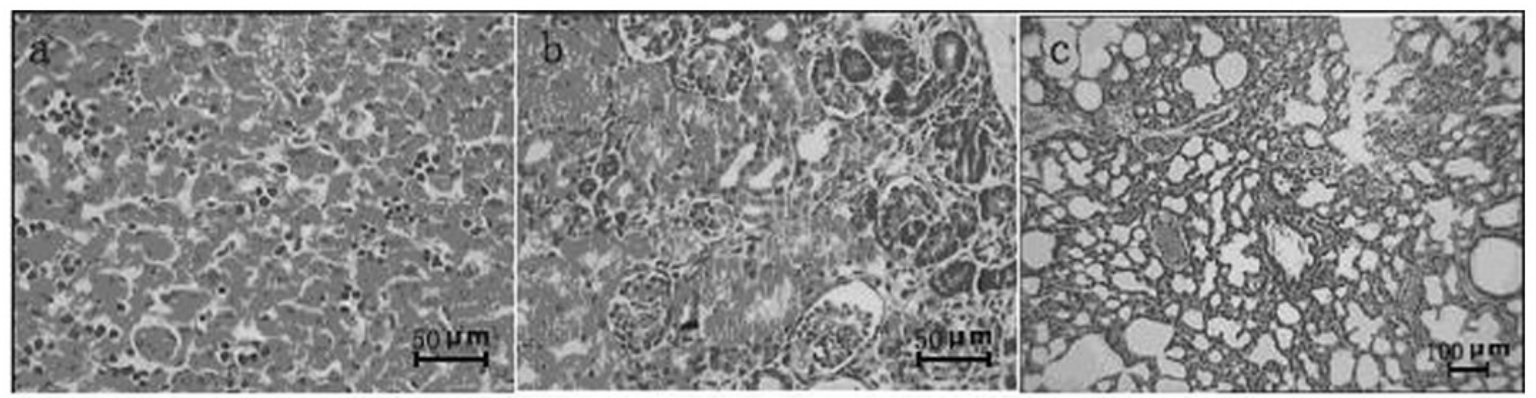

Figure 3. Pathological changes in liver, kidney and lung tissues (hematoxylin and eosin staining). (A) Hepatocytes present with pyknosis and necrosis, as well as inflammatory cell infiltration (magnification, $\mathrm{x} 400$ ). (B) Renal glomeruli are swollen, renal tubules are hydropic and interstitium is hemorrhagic (magnification, $\mathrm{x} 400$ ). (C) The pulmonary alveolus structure is destroyed, and the pulmonary interstitial compartment is thickened and hemorrhagic, with infiltrating inflammatory cells (magnification, $\mathrm{x} 100$ ).

\section{Discussion}

The precise etiological factors and pathogenesis of NEC remain unclear, but certain high risk factors, including premature delivery, artificial feeding, hypoxia and bacterial colonization have been described, and some progress has been made in NEC treatment. Animal models play a significant role in NEC research. Since Barlow et al (13) first adopted a complex method involving artificial feeding, hypoxia and microbial colonization to successfully establish neonatal rat NEC models in 1974, most scholars began to use a modified method including premature delivery, artificial feeding, hypoxia and cold stimulation to establish NEC models. Single factor methods, including the intraperitoneal administration of LPS and hypoxia-reoxygenation have primarily been used in China to establish NEC models.

Hypoxia-reoxygenation is accepted as the primary method of establishing NEC and continues to be utilized. Hypoxia induces the diving reflex, redistribution of systemic blood, mesenteric vasoconstriction, increased resistance, reduced blood supply in the intestinal tract (18), and formation of a variety of substances including oxygen-free radicals, plateletactivating factors (19), interleukin-1 $\beta$, interleukin-6 and tumor necrosis factor (TNF)- $\alpha$ (20), all of which contribute to intestinal tract injury. However, Caplan et al (21) reported that hypoxia-reoxygenation may cause intestinal tract injury, but it hardly induces typical NEC in rats. Furthermore, recent clinical findings have demonstrated that perinatal asphyxia does not enhance the NEC morbidity rate (22-24). Doppler studies of antenatal intestinal blood flow have also not supported a greater likelihood of stage 2 or 3 NEC in infants who had lower blood flows (25). The present study provided similar results, demonstrating only a $20 \%$ NEC morbidity rate and mild intestinal tract injury in the hypoxia-reoxygenation group.

LPS is a significant component in the outer membrane of gram-negative bacteria. It is capable of binding with the CD14/TLR4/MD2 receptor compound and promoting inflammatory cells to secrete various cytokines, thereby causing an intense inflammatory reaction. Various points have been made regarding the effect of the LPS injection for modeling. Certain scholars reported that by LPS injection alone it is 
difficult to establish a successful model of neonatal NEC (26). However, Lu et al (27) suggested that intraperitoneal injection of LPS can result in $87.5 \%$ of neonatal NEC. In our study, LPS administration has been used to successfully establish NEC models, with a morbidity rate of $60 \%$, but this method has numerous limitations. First, LPS is an intermediate product of inflammatory reaction, which results in NEC-like changes of the intestine without consideration of the major risk factors of NEC. Second, as an inflammatory mediator, LPS not only induces intestinal tract injury, but also impairs the liver, kidneys and lungs, which contributes to endotoxemia. In the present study, severe hemorrhagic necrosis of the liver, kidneys and lungs was observed in the LPS group, and in the two cases of mortality the rats may have suffered multiple organ failure as a result of LPS stimulation to the liver and lungs, thereby affecting NEC specificity.

A premature intestinal tract, simulated milk artificial feeding and ischemia/hypoxia are closely related to NEC. Prematurity is clearly one of the main risk factors that predispose to the development of NEC (28), because of the immaturation of anatomical, functional and immunological aspects of the gut. In addition, artificial feeding with simulataed milk is considered to be another risk factor, leading to a NEC morbidity rate greater than that observed with breast milk $(29,30)$. On the one hand, simulated milk contains glucose, fat and protein contents similar to breast milk. However, it lacks epidermal growth factor, platelet-activating factor acetylhydrolase, interleukin-10, secretory immune globulin A, mucoprotein, lactoferrin and probiotic bacteria, which exhibit protective effects (31-33). In contrast, when artificial feeding is used, repeated intragastric administration results in digestive tract injury and bacterial invasion. Rapid and great amounts of feeding aggravate malabsorption of protein and lactose. In addition, bacterial fermentation produces gas, which increases intestinal wall pressure, thereby inducing intestinal mucous ischemia and intestinal tissue injury (34). In the present study, neonatal and mother rats from the hypoxia-reoxygenation and LPS groups were housed in one cage. During NEC induction, the mother rats took care of the neonatal rats, the intestinal mucosal barrier was allowed to gradually develop, and intestinal functions gradually matured. As a result, intestinal tract injury was relatively mild. Nevertheless, feeding with rat milk may result in a serious impairment of other vital organs, as well as mortality, since it does not directly protect these organs. The intestinal injuries observed in the two cases of mortality were extremely serious. NEC-induced death was presumed in these cases, and from this we may consider that the incidence of NEC may have been as high as $90 \%$ in group A.

An ideal NEC animal model should simulate human conditions, including etiological factors, pathogenesis and clinical manifestations, and it should be characterized by a short gestational period, reproducibility, high specificity, and a simple, feasible model establishment with a high success rate. The present study simulated the high-risk factors of NEC using simulated milk artificial feeding, hypoxia and cold stimulation. Results revealed that the majority of the neonatal rats presented with typical NEC pathological changes. Although the use of LPS and hypoxia-reoxygenation for NEC induction does not correspond to NEC etiological factors, the morbidity rate is low, but maintains specificity. In conclusion, this method involving premature delivery, artificial feeding of simulated milk, hypoxia and cold stimulation is considered an ideal method for establishing animal models of NEC.

\section{Acknowledgements}

Supported by grants from the Guangdong Natural Science Fund Committee (No. 8151012001000002).

\section{References}

1. Hunter CJ, Upperman JS, Ford HR and Camerini V: Understanding the susceptibility of the premature infant to necrotizing enterocolitis (NEC). Pediatr Res 63: 117-123, 2008.

2. Schnabl KL, VanAerde JE, Thomson ABR and Clandinin MT: Necrotizing enterocolitis: A multifactorial disease with no cure. World J Gastroenterol 14: 2142-2161, 2008.

3. Holman RC, Stoll BJ, Clarke MJ and Glass RI: The epidemiology of necrotizing enterocolitis infant mortality in the United States. Am J Public Health 87: 2026-2031, 1997.

4. Meinzen-Derr J, Morrow AL, Hornung RW, Donovan EF, Dietrich KN and Succop PA: Epidemiology of necrotizing enterocolitis temporal clustering in two neonatology practices. J Pediatr 154: 656-661, 2009.

5. Puiman P and Stoll B: Animal models to study neonatal nutrition in humans. Curr Opin Clin Nutr Metab Care 11: 601-606, 2008.

6. Sangild PT: Gut responses to enteral nutrition in preterm infants and animals. Exp Biol Med (Maywood) 231: 1695-1711, 2006.

7. Gellén B, Kovács J, Németh L, Németh P, Vagvölgyi J and Bari F: Vascular changes play a role in the pathogenesis of necrotizing enterocolitis in asphyxiated newborn pigs. Pediatr Surg Int 19: 380-384, 2003

8. Cadir FO, Bicakci U, Tander B, Kilicoglu-Aydin B, Rizalar R and Ariturk E: Protective effects of vitamin $\mathrm{E}$ and omeprazole on the hypoxia/reoxygenation induced intestinal injury in newborn rats. Pediatr Surg Int 24: 809-813, 2008.

9. Gonzalez-Crussi F and Hsueh W: Experimental model of ischemic bowel necrosis: The role of platelet-activating factor and endotoxin. Am J Pathol 112: 127-135, 1983.

10. Sun XM and Hsueh W: Bowel necrosis induced by tumor necrosis factor in rats is mediated by platelet-activating factor. J Clin Invest 81: 1328-1331, 1988.

11. Torimoto K, Sato N, Okubo M, Yagihashi A, Wada Y and Hara I: Development of multiple necrotizing enteritis induced by tumor necrosis factor like cytokine from lipopolysaccharide-stimulated peritoneal macrophages in rats. Am J Pathol 137: 1103-1111, 1990.

12. Chung DH, Ethridge RT, Kim S, Owens-Stovall S, Hernandez A and Kelly DR: Molecular mechanisms contributing to necrotizing enterocolitis. Ann Surg 233: 835-842, 2001.

13. Barlow B, Santulli TV, Heird WC, Pitt J, Blanc WA and Schullinger JN: An experimental study of acute neonatal enterocoliti - the importance of breast milk. Pediatr Surg 9: 587-594, 1974.

14. Guven A, Gundogdu G, Uysal B, Cermik H, Kul M and Demirbag S: Hyperbaric oxygen therapy reduces the severity of necrotizing enterocolitis in a neonatal rat model. J Pediatr Surg 44: 534-540, 2009.

15. Whitehouse JS, Xu H, Shi Y, Noll L, Kaul S and Jones DW: Mesenteric nitric oxide and superoxide production in experimental necrotizing enterocolitis. J Surg Res 161: 1-8, 2010.

16. Le Mandat Schultz A, Bonnard A, Barreau F, Aigrain Y, Pierre-Louis C and Berrebi D: Expression of TLR-2, TLR-4, NOD2 and pNF-kappaB in a neonatal rat model of necrotizing enterocolitis. PLoS ONE 2: e1102, 2007.

17. Auestad N, Korsak RA, Bergstrom JD and Edmond J: Milk-substitutes comparable to rat's milk; their preparation, composition and impact on development and metabolism in the artificially reared rat. Br J Nutr 61: 495-518, 1989.

18. Scholander PF: The master switch of life. Sci Am 209: 92-106, 1963.

19. Akisu M, Küllahçioğlu Girgin F, Baka M, Hüsseyinov A and Kültürsay N: The role of recombinant human erythropoietin in lipid peroxidation and platelet-activating factor generation in a rat model of necrotizing enterocolitis. Eur J Pediatr Surg 11: 167-172, 2001. 
20. Kumral A, Yesilirmak DC, Tugyan K, Baka M, Hüsseyinov A and Kültürsay N: Activated protein $C$ reduces intestinal injury in an experimental model of necrotizing enterocolitis. J Pediatr Surg 45: 483-489, 2010

21. Caplan MS, Hedlund E, Adler L and Hsueh W: Role of asphyxia and feeding in a neonatal rat model of necrotizing enterocolitis. Pediatr Pathol 14: 1017-1028, 1994.

22. Yu VY, Joseph R, Bajuk B, Orgill A and Astbury J: Perinatal risk factors for necrotizing enterocolitis. Arch Dis Child 59: 430-434, 1984.

23. Guthrie SO, Gordon PV, Thomas V, Thorp JA, Peabody J and Clark RH: Necrotizing enterocolitis among neonates in the United States. J Perinatol 23: 278-285, 2003.

24. Lee JS and Polin RA: Treatment and prevention of necrotizing enterocolitis. Semin Neonato 18: 449-459, 2003.

25. Kirsten GF, van Zyl N, Smith M and Odendaal H: Necrotizing enterocolitis in infants born to women with severe early preeclampsia and absent end-diastolic umbilical artery doppler flow velocity waveforms. Am J Perinatol 16: 309-314, 1999.

26. Gonzalez-Crussi F and Hsueh W: Experimental model of ischemic bowel necrosis. The role of platelet-activating factor and endotoxin. Am J Pathol 112: 127-135, 1983.

27. Lu H and Xie XD: Dynamic changes of epidermal growth factor and its receptor in neonatal rats with intestine injury. Chinese Journal of Perinatal Medicine 11: 173-176, 2008
28. Afrazi A, Sodhi CP, Richardson W, Neal M, Good M and Siggers R: New insights into the pathogenesis and treatment of necrotizing enterocolitis: Toll-like receptors and beyond. Pediatr Res 69: 183-188, 2011.

29. Lucas A and Cole TJ: Breast milk and neonatal necrotizing enterocolitis. Lancet 336: 1519-1523, 1990.

30. Dvorak B, Halpern MD, Holubec H, Dvorakova K, Dominguez JA and Williams CS: Rat milk decreases necrotizing enterocolitis in a rat model. Adv Exp Med Biol 554: 471-473, 2004.

31. Walker WA: Breast milk and the prevention of neonatal and preterm gastrointestinal disease states: a new perspective. Zhonghua Min Guo Xiao Er Ke Yi Xue Hui Za Zhi 38: 321-331, 1997.

32. Dvorak B, Halpern MD, Holubec H, Dvorakova K, Dominguez JA and Williams CS: Maternal milk reduces severity of necrotizing enterocolitis and increases intestinal IL-10 in a neonatal rat model. Pediatr Res 53: 426-433, 2003.

33. Walker WA: Breast milk and the prevention of neonatal and preterm gastrointestinal disease states: a new perspective. Zhonghua Min Guo Xiao Er Ke Yi Xue Hui Za Zhi 38: 321-331, 1997.

34. Okada K, Fujii T, Ohtsuka Y, Yamakawa Y, Izumi H and Yamashiro Y: Overfeeding can cause NEC-like enterocolitis in premature rat pups. Neonatology 97: 218-224, 2010. 